\title{
Hybrid Force-Based Model to Simulate Behavior of Human Crowd in Panic Situations
}

Ammar Alnahhas, Elisaar Barri

Faculty of Information Technology Engineering, Damascus University

Faculty of Informatics and Communication, Arab International University

Syrian Arab Republic

a.alnahhas@damasuniv.edu.sy, eng.a.alnahhas@gmail.com

elisaarbarre.1997.sy@gmail.com

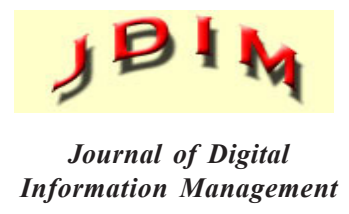

ABSTRACT: Modelling human behavior is important as it helps preparing for the situation of emergency to save lives. In this paper we present new methods to simulate crowd evacuation phenomena by using a physical model. We focus on applying more effective forces focusing on Spring-Force as general forces affecting agents as well as repulsive danger-force, in addition, we propose a new algorithm for an interaction behavior. We implement our algorithm in a real-world application which shows that our model provides results approaching reality and is promising compared to the other models.

Subject Categories and Descriptors

I.6 [Simulation and Modelling]: I.2 [Artificial Intelligence]; Intelligent Agents

General Terms: Simulation, Intelligent Agents

Keywords: Simulation, Force-based Model, Human Crowd Simulation

Received: 15 September 2018, Revised 29 October 2018, Accepted 20 November 2018

Review Metrics: Review Scale - 0/6, Review Score - 4.65, InterReviewer Consistency - 72.2\%

DOI: $10.6025 / \mathrm{jdim} / 2019 / 17 / 1 / 25-33$

\section{Introduction}

Crowd Phenomena have attracted many researchers in the recent years, tending to understand and analysis its details aiming at finding the most realistic models to build a simulation as a useful tool in a lot of fields such as entertainment (computer games industry), robotic [1], education, training(for military and police), and in architectural design both for everyday use and for emergency evacuation conditions.

Researchers are facing many challenges in crowd simulation such as modeling group structures which play important roles in affecting crowd behavior, for instance [2] defined a unified framework to systematically model the different aspects of group structures in pedestrian crowds. Also interactive navigation and path finding are both another important challenge where researchers try to model human spatial navigation such as [3], and [4] which use agent-based and predictive approach, whereas in [5] which presented a novel mathematical formulation for generating energy-efficient trajectories based on biomechanical principles for guiding agents in crowd simulation. Other challenges like simulating large and dense crowds in real time [6], and integrating psychological model into a crowd simulation system [7], and also collision avoidance like [8] which will be discussed in detail later.

In this work we focus on collision avoidance and navigation behaviors specifically in the emergency situation which is important especially because of the need of providing solid evacuation system in areas where large number of people gather. This requires understanding crowd behaviors 
and dynamics during emergency situations.

According to Helbing [9] - who is one of the investigators in this field-some characteristics and features of escape panics are:

1. People move or try to move considerably faster than normal.

2. Individuals start pushing, and interactions among people become physical in nature.

\section{At exits arching and clogging are observed.}

4. Moving and in particular passing of the bottleneck frequently becomes uncoordinated.

5. People tend to show herding behaviors to do what other people do.

So, inspired by the actions that are noticed in crowd evacuation and starting from Helbing findings, we try to approximate that actions into physical phenomena such that we consider the people escaping from danger sources as particles that are pushed back by repulsive force in a charged field. Besides the attraction force that pull people to the target point (either the exit or group of people) could be considered as the restoring force of a stretched virtual spring.

This paper is structured as follows: we present related researches in Section 2. Then in Section 3 we describe our model in details, the forces used and the algorithm used, moving to Section 4in which we show the practical simulation and some results we notice, finally in Section 5 we suggest the future work and conclude the paper.

\section{Related Work}

As we mentioned before crowd evacuation has been investigated for years to find more accurate models that describe human behavior in panic situations and thus provide simulated experiments rather than real-life one that could be dangerous for humans.

Generally we can classify related works into two distinct classes macroscopic and microscopic models [10]:

Macroscopic Continuum Models which describe collective properties without distinguishing individual particles, instead the state of the system is described by densities. The first modelling attempt is Hughes [11] who defined the crowd as a "thinking fluid" and described the time evolution of its density using a scalar conservation law. Another work [12] analyzed numerically two macroscopic models of crowd dynamic which are Hughes and a new model that they used it to study the evacuation of pedestrians from a room through a narrow exit.

In Microscopic Models, each agent is represented separately. Such an approach allows to introduce different types of agents with individual properties, as well as issues like route choice. Many models of this type discussed evacuation phenomena:

\section{- Cellular Automata Models [10]}

These models are discrete in the three basic variables which describe the system, namely space, time, and state variable. Although CA models describe the properties of crowd fairly well, however the discretization of space is not always possible in sensible way.

\section{- Force-Based Models}

In contrary with CA these models are defined in continuous space. they follow Newtonian dynamic and consider that the forces defining the equation of motion are split into driving force and repulsive force. The driving force models the intention of agents to move to a certain destination and walk with a desired velocity, and the repulsive force models the collision avoidance performed by agents.

The origin of force-based modeling can be traced back to the beginning of the 1950s [13], later many models was developed such as social force model by Helbing and colleagues, they explained that this force is not exerted by the environment on a pedestrian's body. It is rather a quantity that describes the concrete motivation to act [14]. Then they study this force in crowd evacuation dynamic[9] and noticed collective phenomena in panic situation as Faster-Is-Slower effect, herding and ignorance of available exits, transition to Stop-and-Go waves, etc. social-force model is capable of describing the self-organizing of several observed collective effects of pedestrian behavior very realistically.

Another example of force-based models is GCFM (the Generalized Centrifugal Force Model) which is different in the geometrical shape of modeled pedestrians [13].

\section{-Agent-Based Models [15]}

$A B M$ are one of the numerical methods useful to simulate the activities and interactions of individual or collective agents. This technique has been used to study crowd evacuation in various situations and to simulate it since the early of 2000 [15]. Pedestrian is treated as autonomous decision-making agents and each one of them makes decision based on the interactions with other agents and surrounding environment. Comparing with CA Models, $\mathrm{ABM}$ are more computational time consuming. Examples of these models are: [16] which is a work to create an efficient, agent-based, individual-centered model of crowd evacuation that incorporates pushing force and injuries into the model using Kirchner model. Others [17] create algorithm that is an extension of the Boids simulation method - which is developed by Craig Reynolds in 1986 with additional rules.

\section{- Discrete Element Method}

DEM is a set of modeling techniques and equations specifically used to solve engineering problems. It models 
the motion and mechanical interactions for each particle in physical problems. As CA, DEM is defined in discrete space. In this method the contact forces are usually calculated by "virtual" springs at the contact points. Also, the forces acting on particles are summed up and as in force-based model Newton's equation of motion is practiced to obtain acceleration, velocity and the displacement of each particle to update the new position of the particles at the next time step.

As the study [15], the earliest research on evacuation simulation integrated with DEM is conducted by Kiyono et al. in 1996who found in his research that the parameters such as spring constant, driving force of human and walking velocity are obtained from experiments. In 1998, Kiyono et al.[18] had modified his work in 1996 by incorporating an algorithm in which element can avoid collision with other elements.

Another work on this field is [19] which study how the desired velocity affects the throughput of the exit by using the DEM.

By the other hand In order to create a realistic and believability crowd simulation, collision avoidance is one of the essential issues that we have considered, in work [20] they give an overview on collision avoidance in crowd simulation such as Loscos et al.(2003) which uses collision map and grid system to do the collision detection in specific by comparing the direction of each agent, the velocity factor and the distance between the agents. Another research by Sakuma et al. (2005) which suggested that the environmental information should be stored in virtual memory and when the collision detected they either follow the rule urgent avoidance or smooth avoidance, depending on the existence of other agents if is detected in the critical area of personal space. Another work [3] used the "Velocity Obstacles" method as a solution for collision avoidance problem which assuming that the other agents are dynamic obstacles whose future motions are predicted as linear extrapolation of their current velocities. And the work [8] which used visionbased approach.

Our method is slightly different from the previous methods, where we define it in continuous space but we use the idea of discrete space into regions as a data structure. We use linear equation of motion of each agent and define two circle fields for each agent that are vision field and personal field which ease the process of navigating and collision avoidance in a less time complex manner. Besides, navigation algorithm that we provide simulates the natural behaviors of scared people, in specific the direction of an agent is affected by not just external forces but also by internal one, because people are self-driven particles and can make decisions from personal views, and this was not considered by all of the previous studies. We show that using the force of spring with behavior algorithms improves the simulation results and provides new breakthrough in this field.

\section{Our Model}

People in panic situation are faci-ng forces which make them move to the next specific position. That forces which affect the agents are both general forces and an internal force. General forces are the environment specifically which are:

\section{Danger-Force that repulsing agents away.}

2. Exit-Force that attracts agents by the restoring force of virtual spring.

3. Flock-Force that attracts agents by the restoring force of virtual spring as well.

Whereas the internal force is the force related to collision avoidance.

The update motion equation of each agent is defined by Newton's Second Law as:

$$
\vec{P}_{n}=\vec{P}_{o}+\frac{1}{m_{i}}\left[\Sigma \vec{F}_{r}+\Sigma \vec{F}_{e}+\Sigma \vec{F}_{f}+\Sigma \vec{F}_{c s}\right]
$$

Where $\vec{F}$ denotes the Danger-Force, $\vec{F}_{e}$ Denotesthe ExitForce, $\vec{F}_{f}$ Denotes the Flock-Force, $\vec{F}_{c s}$ Denotes the Collision Spring-Force, $\vec{P}_{n}$ is the new position and $\vec{P}_{o}$ is the position in previous time step in the simulation.

On the other hand, we have to describe the real behavior of agents, for instance Herding behavior would appear depending on what agent see in her vision field, so we try to build an algorithm that approach the real behavior for vision.

Besides real people walk/run in the direction of his aim point, so before changing agent's position we have to check for the angle between his vision vector and effecting forces vector if it is big enough to make turn before transform, or else make the agent moving and turning around by the small angle.

Finally, collision avoidance is also an important behavior that we should take it care.

\subsection{Affecting Forces \\ 3.1.1 General Forces Danger-Force:}

In evacuation cases with existence of danger, people try to go far from them as fast as they can and whenever they are close to danger they acquire big force that increase their acceleration. So, we consider that the danger source is positive isolated charge which its electric field lines are simply a sequence of evenly-spaced, radially directed lines pointed outwards from the danger source, so the agents as a particles will follow the tangent of this electric field line by repulsive force in form:

$$
F_{r^{i}}=-\frac{Q}{d_{i}}
$$


Where $Q$ is the intensity of danger which depends on the nature of danger source such as fire, burst, or electrical fault, etc. And $d_{i}$ is the distance of agent I from danger source. In practical experiment we found that the linear distance $d_{i}$ is more appropriate than quadratic $d_{i}^{2}$.

\section{Aim Forces:}

Agents in panic situations get anxious and nervous so their motion seems to be chaotic attempting to find the exit. Indeed, they lose the ability of awareness to their environment so they become independent and tend to follow a group of agents until finding the exit.

This behavior is called Herding which is the main reason of disastrous events, we simulate it by assuming that each agent is attracted to the direct nearest group by virtual stretched spring which its free side is connected to that agent and its equilibrium point is inside the group's position. The restoring force of this spring is in form:

$$
F_{f^{i}}=-k_{f} \cdot \Delta x_{i}
$$

Where $k_{f}$ is a constant factor characteristic of the Flockspring: its stiffness, and $\Delta x_{i}$ is the distance of agent $i$ from the group position.

However, once the agent finds an exit they will stop tracking other agents and will be connected to virtual stretched Exit-Spring that attracts them to it. As in FlockSpring, the equilibrium point of Exit-Spring is inside the exit position and its restoring force is:

$$
F_{e^{i}}=-k_{e} \cdot \Delta x_{i}
$$

Where $k_{e}$ is a constant factor characteristic of the Exitspring: its stiffness.

\subsubsection{Internal Forces (Interactions)}

Collision avoidance is the main interactions between agents in panic cases. Where people try to keep a distance from each other. As in DEM method, in order to simulate this behavior, we assume that each agent has a personal range in a circular form which its center is positioned on agent's circumference at the front side of agent, with virtual springs inside it.

The algorithm goes along two steps:

Collision Detection: The agent $i$ checks if there are agents inside his personal range. Dividing the environment helps us here because agent checks just for both all agents inside his zone (square) and all the agents in neighboring zones which intersect with personal range of agent $i$. then for each detected agent $j$ he calculates the distance $\Delta x_{i j}$ between him and agent $j$, to predict one of two cases that are Physical Interaction and Collision Resistance which will be described next:

In Case of Collision Resistance: This happens when
$\Delta x_{i j}>0$ which means that both agents haven't collided with each other yet. As what is observed from the human behavior in this case, the person tries to reduce his velocity and changes the direction of his motion, thus we propose that the personal range of an agent has virtual springs which they are connected with the center of the agent and the length of each of them is equal to the radius of his range. So the presence of agent $j$ inside the personal range of agent $i$ leads to compression a spring forming restoring force $F_{c s}$ :

$$
F_{c s^{i}}=-k_{s c} \cdot \Delta x_{i j}
$$

Where $k_{s c}$ is a constant factor characteristic of the Collision-spring i.e. Its stiffness. This force affecting on agent $i$ assuming that the constant side of this spring is connected to agent $j$.

In case of Physical Interaction: This case happens once $\Delta x_{i j}<=0$, so to resolve the physical collision between agent $i$ and agent $j$ we used the equation which expresses conservation of momentum that helps to find the velocities of agents after collision as following:

$$
\begin{aligned}
V_{i} & =\frac{m_{i} U_{i}+m_{j} C_{R}\left(-U_{i}\right)}{m_{i}+m_{j}} \\
V_{j} & =\frac{m_{i} U_{i}+m_{i} C_{R}\left(+U_{i}\right)}{m_{i}+m_{j}}
\end{aligned}
$$

Where $V_{i}$ and $V_{j}$ are the velocity after collision of agent $i$ and agent $j$, and $U_{i}$ and $U_{j}$ are the velocity before collision of agent $i$ and agent $j$, respectively. Is the Coefficient of Restitution that equal to $C_{R}=\left(V_{j}-V_{i}\right) / U_{i}$, assuming that agent $j$ is constant at the time of collision.

We used this measure $C_{R}$ because human collision is not elastic; there are always some dissipation depending on the feature of person's body, so in our simulation we used $\left(C_{R}=0.3\right)$.

\subsection{Behaviors' Algorithms}

As mention briefly before, we divide the space into virtual zones where each cell contains group of people in which they surely collide with each other, so each agent knows the zone that he belongs to. For the zone ${ }_{n m}$ we defined its direct neighboring zones:

$$
\begin{gathered}
\text { zone }_{n-1 m-1}, \text { zone }_{n-1 m}, \text { zone }_{n-1 m+1}, \text { zone }_{n m-1}, \text { zone }_{n m+1} \\
\text { zone }_{n+1 m-1}, \text { zone }_{n+1 m}, \text { zone }_{n+1 m+1},
\end{gathered}
$$

\section{Vision behavior}

We assume that each agent has his own vision field that describes what he can see, this field is in circle form where agent is on its circumstance looking to its center point and can see all the space of this circle field. Because the vision of normal human is bounded by existence of 
objects, we considered its radius equals to the diameter of the square room that he/she is inside.

Intuitively, scared people in panic situations will naturally follow the first safest thing they will find, so depending on the previous assumptions, the algorithm of vision behavior that determines where to go would be for each agent as:

1. Calculating the density of each direct neighboring zones of the agent's zone which are in his/her vision field. By geometric calculations we can do that.

\section{Then we have the following cases:}

- If the exit is in one of that zones he/she will follow directly.

- Else agent will follow the zone with the most density (Herding behavior)

- In case all densities values are zeros, agent will continue searching in the next neighboring zones which are the neighbors of direct neighbors as in figure 1.

- If agent is lonely he/she will just turn around.

\section{Collision Avoidance}

As in "vision field", agents have another field named "personal field" which is the region that others are not allowed to be there, but the size of this field is equal to twice agent's size.
Before changing the old position of agents, they will check for collision, first in collision detection phase agent will search in his/her personal field for agents that may collide with them, in the same method as described in vision algorithm we search for an agent but in this case searching will be just for the direct neighboring zones of the agent's zone.

Next in collision avoidance phase the agent will change his/her path depending on the sum of all springs forces from each colliding agent and resolve the physical collision if happened (push forces).

\section{Results}

To test our model, we simulate a crowd trying to escape a great burning room where we assumed that there are no constant obstacles other than walls. And the environment is two dimensional. Our application is designed using Unity game engine, and the simulation shows good results in comparing with other works. Using the force of spring as general forces in addition to collision force increases the velocity of agents which presents more real behavior, for instance in the Figure 3 we try to run a simulation with 150-agents in a huge room contains a fire near the door which is very small comparing with the size of room. We set the intensity of fire $Q=100000$ and the constant of Exit_force $k_{e}=50$, the result was people

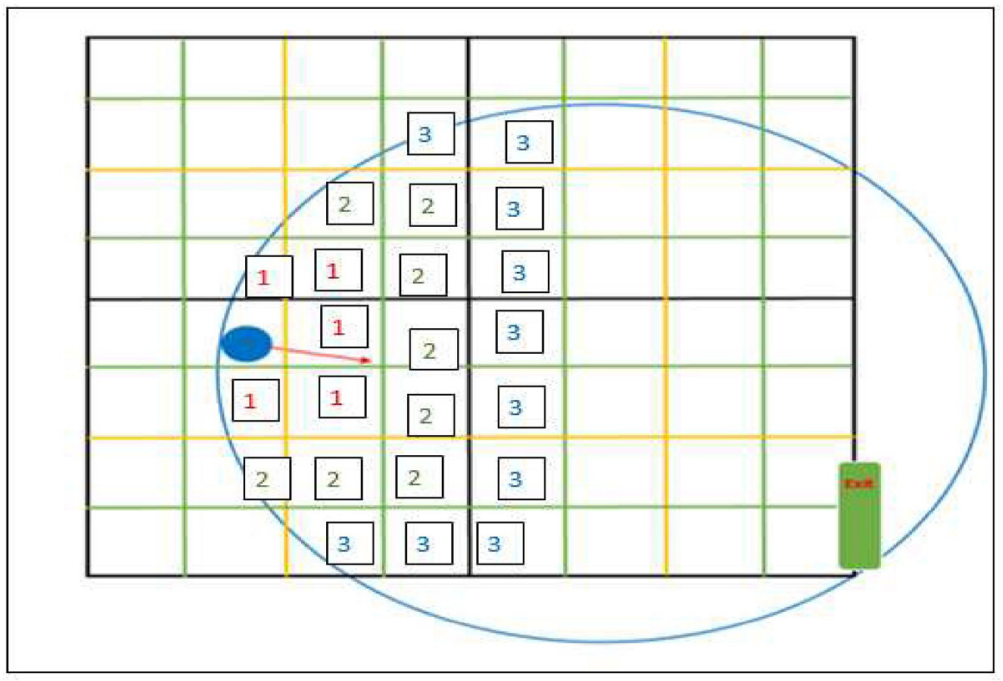

Figure 1. Algorithm of vision behavior

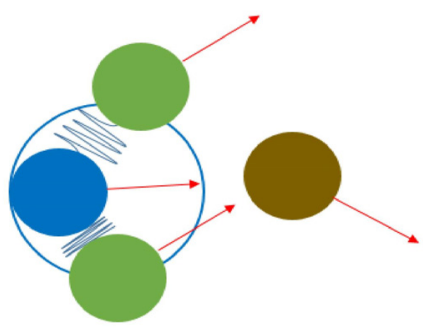

Figure 2. Collison detection. The agent with blue color has already detected 2 agents which are green agents in his personal field, then he will be affected by 2 forces produced by the 2 virtual springs. The agent with brown color is still not detected yet. Red arrows refer to direction of velocity of agents 


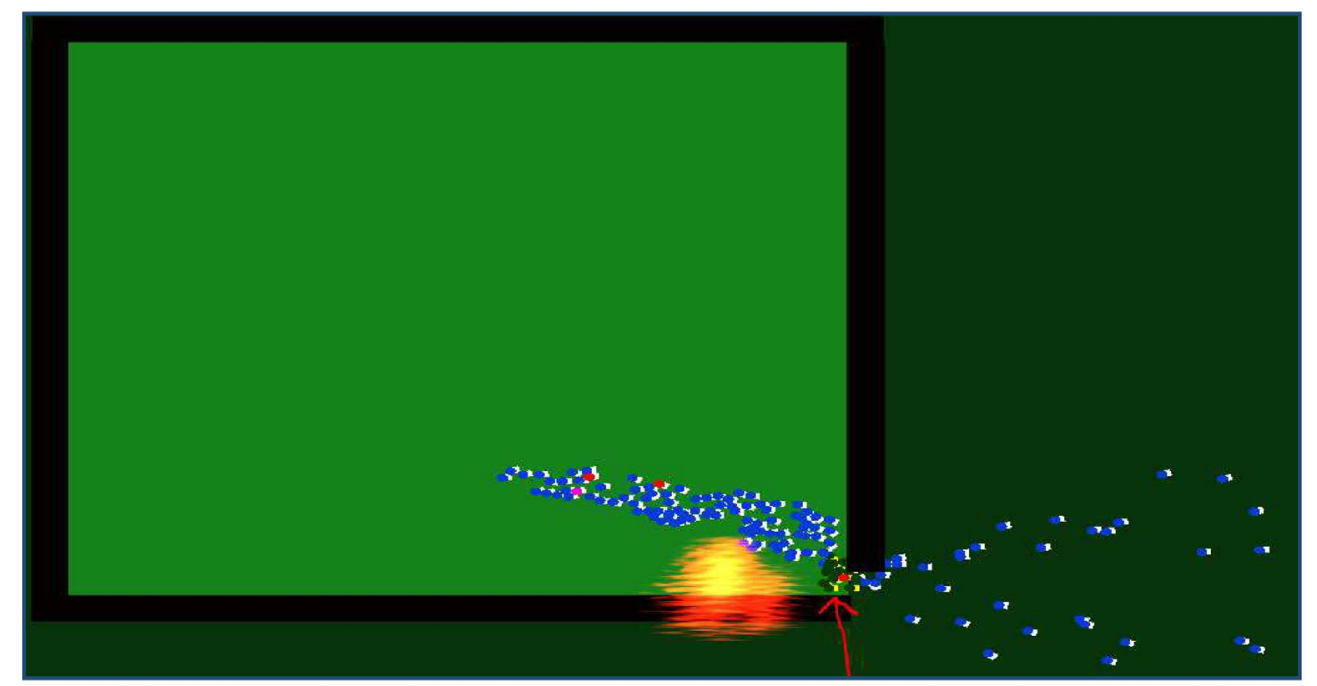

Figure 3. Arching and lines formation near the exit

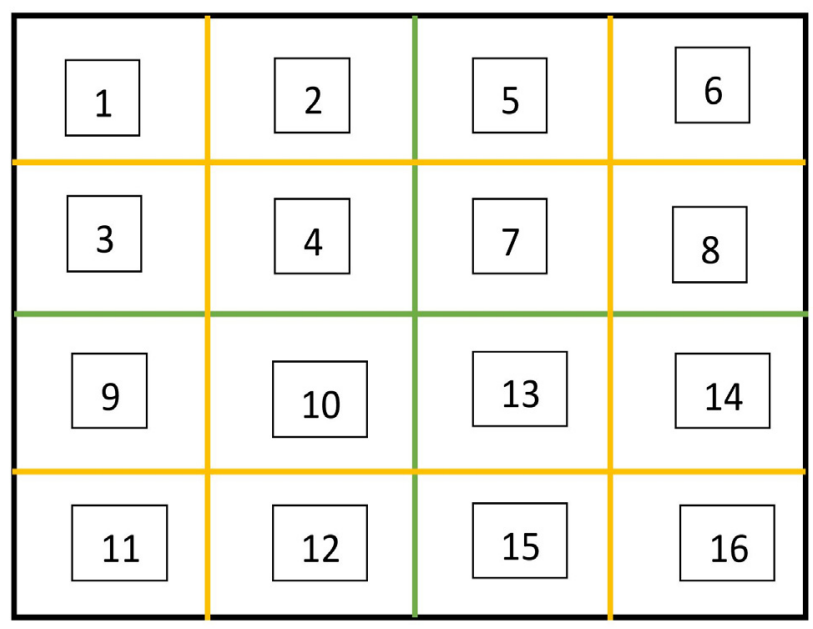

Figure 4. Regions in the room

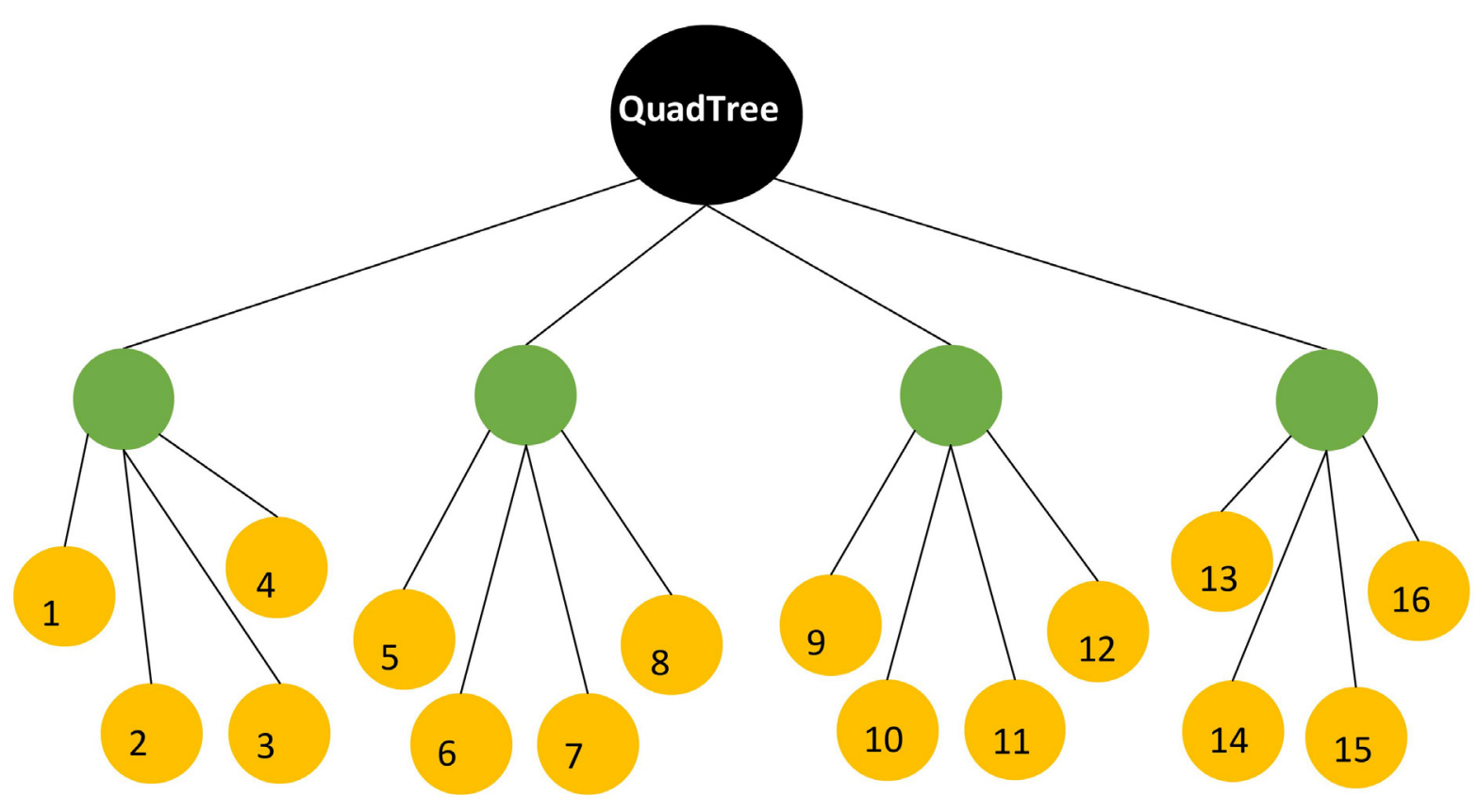

Figure 5. QuadTree illustrates zones of the room in Figure 4 
escaped during 3-minutes, and when we make the fire far from the door in the Figure 6 , the time of escaping become 41-seconds.

In other experiment (Figure 7) we make the room small and contain 25-agents with a fire far from the door, and the time for escaping was 1 minute and 1.82 seconds.

\section{The use of QuadTree}

It is worth mentioning that we used QuadTree to divide the space into zones, as it is an efficient hierarchical spatial data structure. We used complete QuadTree which its leaves are the zones of the space, so it helps to improve collision avoidance technique specifically collision detection as we mentioned before. And also it helps in reduce the time of searching for objects in the space (other agents - constants) since its complexity is $0\left(\log _{4}(m)\right)$.

Then when updating the position of an agent for each none, depending on our algorithms we need to search for at most 8 neighbor zones in the tree in complexity of $0\left(\log _{4}(m)\right)$ so the final complexity is $0\left(n \cdot \log _{4}(m)\right)$.

\section{Behaviors Analysis}

From the experiments we did, we noticed the following formations:

- Lines as a result of herding behavior.

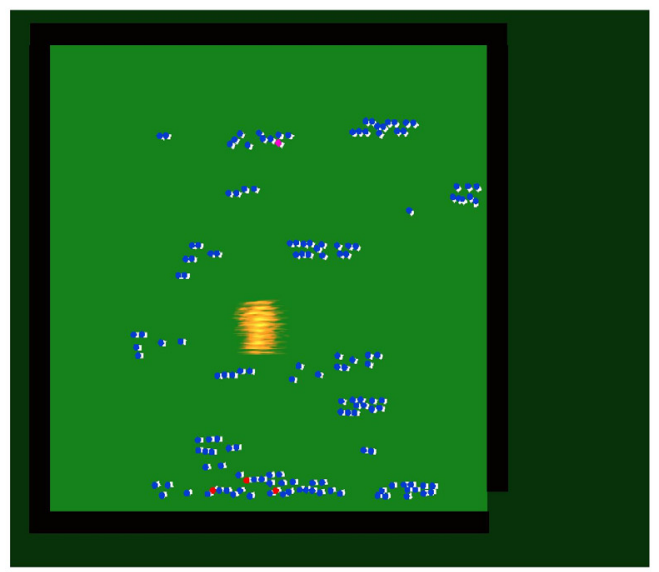

a) After 1.51 minutes

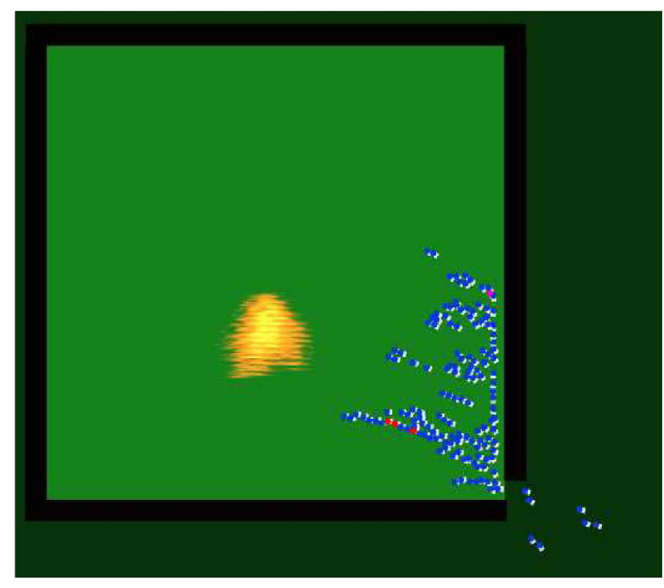

c) After 14.91 minutes
- Waves at the exit point as a result of collision forces.

- Arching at the exit.

First of all, from the Figure 6 which shows the simulation situations we can observed the alternation between these formations during evacuation scenario, where in (a) at the time $t=1.51$, the danger source starts affecting on agents and over the time because of herding behavior we can see the lines formation (b, c), then at the time $t=23.03$ groups of agents increase at the door which cause increasing of the collision forces between them causing In (d) to form the stop-and-go waves which in its turn push agents back and form the lines again at time $t=29.30$ (e, f).

We noticed also in this test that it is not necessary that agents close to the exit be able to get first because of the waves and the push forces as we said before, for instance the agents with red color near the exit get out after the time $=38.85$ minutes.

By the other hand in the test illustrated in the Figure 7 agents with yellow color are still searching for either the exit or other people to follow, but agents with red color have seen the exit and become effecting by the exit force and collision avoidance forces.

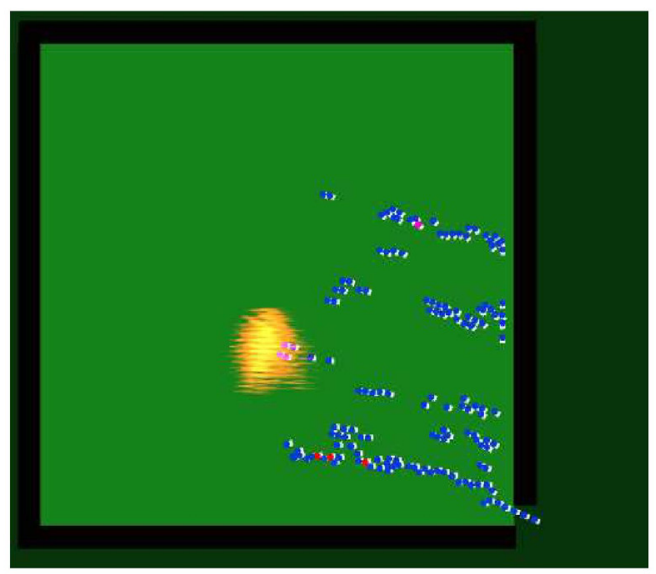

b) After 10.35 minutes

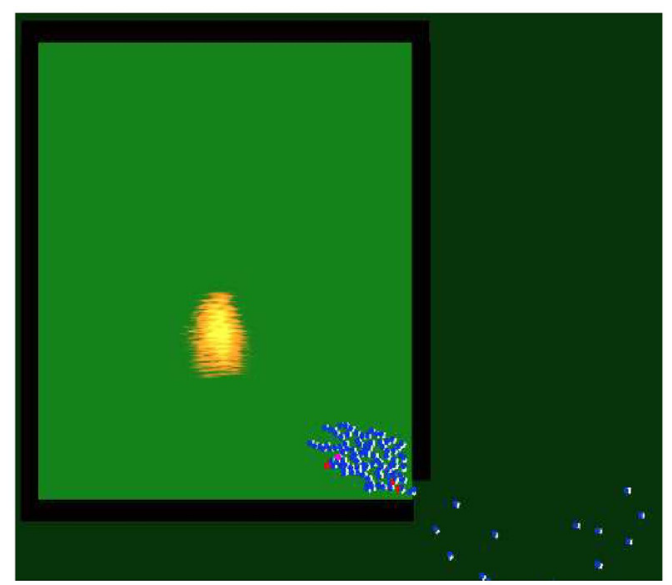

d) After 23.03 minutes 


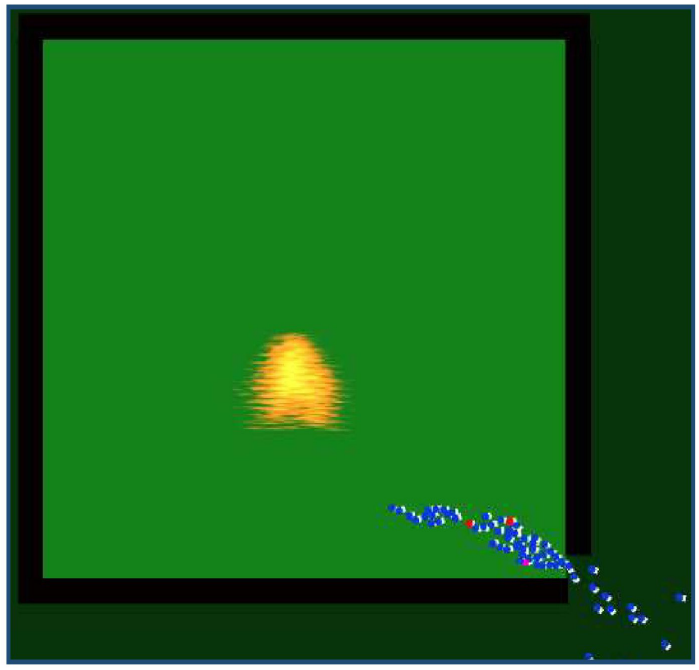

e) After 29.30 minutes

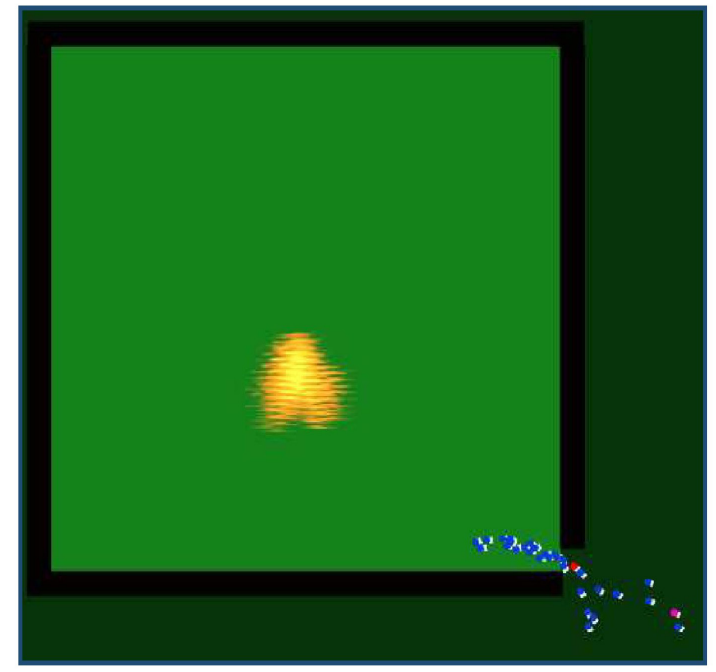

f) After 38.85 minutes

Figure 6. Rresult of a simulation. Agents with red color were near to the exit at the first of the simulation (a), despite that we noticed that they are with the last agents could evacuate out of the room (f)

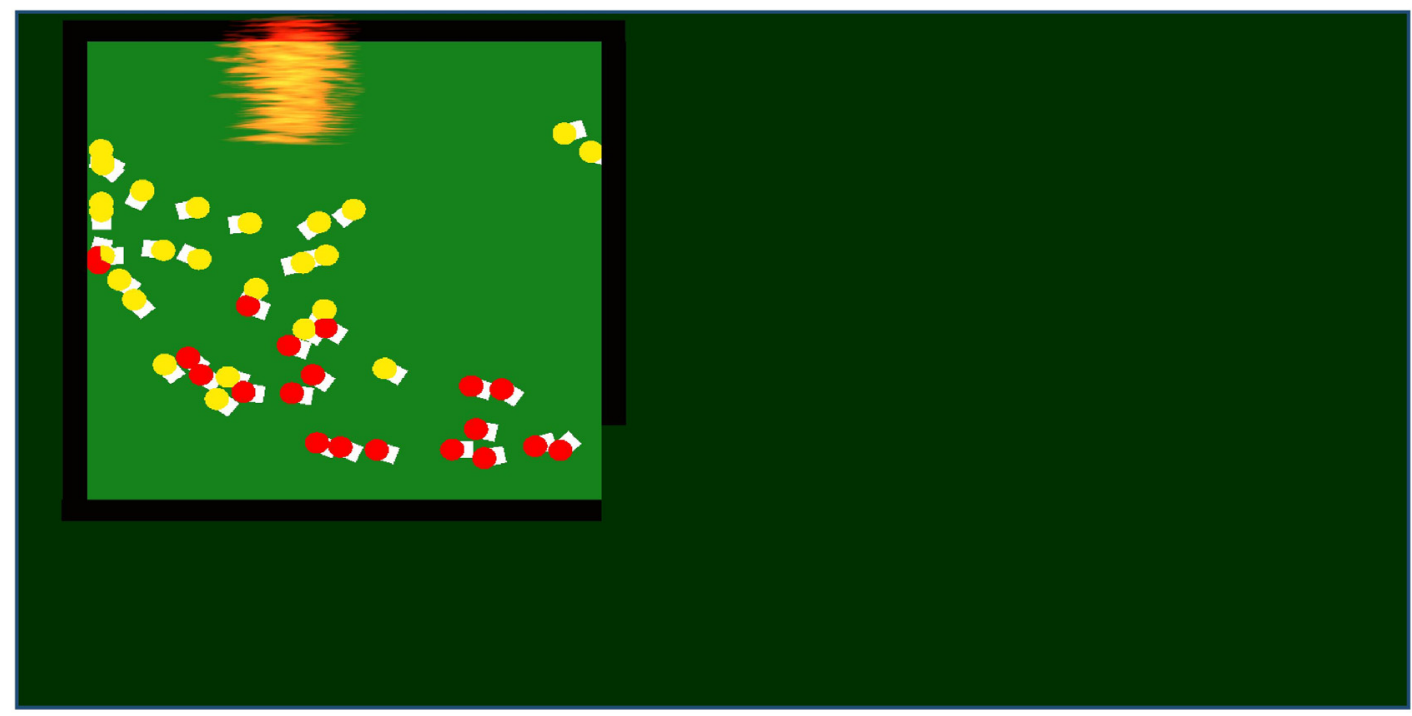

Figure 7. Result of a simulation. Where red agents have seen the door, yellow agents can see just other agents.

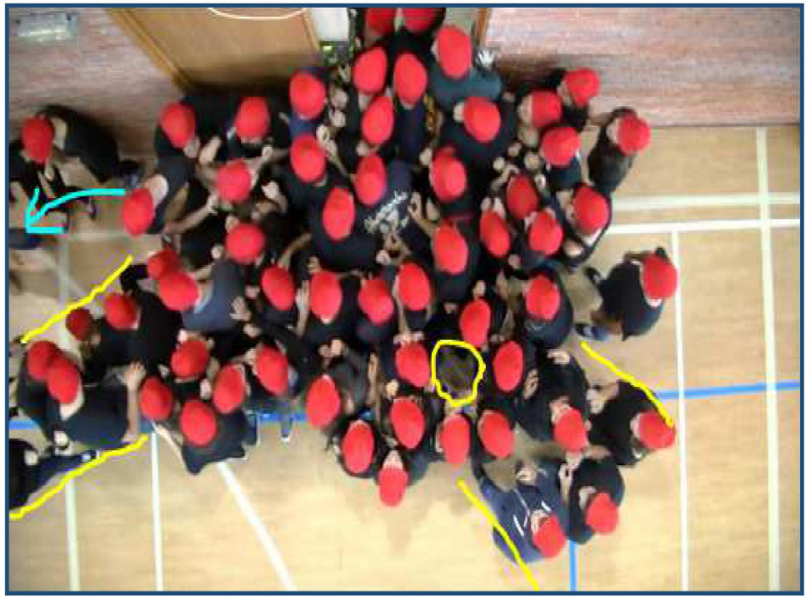

a) Shows the push back behavior, and lines

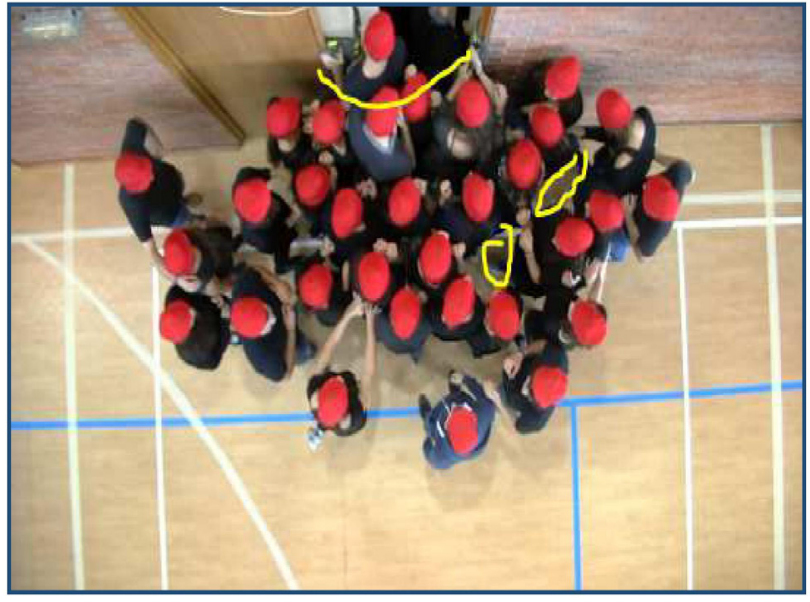

b) Shows the arch near by the exit

Figure 8 . Real video of crowd evacuation. 
For the purpose of measuring the ability of our model to simulate real human behaviors and to compare result with previous work, we compare it with real video of crowd evacuation from a bottleneck with high competitive situation. We noticed from (figure 6) that using spring force either as internal force (collision resistance) or external force (exit attraction), shows the same behaviors that are presented from video in the (figure 8). Where we can see in (figure 8 - a) the gaps between evacuees and the stop and go waves that are caused from the internal push. In (figure 8 - b) we can see the lines and arches near the exit. Without using spring forces that are not used in previous researches the simulation gets inaccurate result compared to real situations, as distance between agents are not realistic and agents tend to be close to each other leaving large vacuum areas which is not present in real situations, and that's what our model solves.

\section{Conclusion}

In this paper we showed a new method to model the behavior of human crowds in panic situations, we showed how the model represents the factor of the environment as a physical forces, we viewed the proposed algorithm which is implemented in a real world application, comparing the results shows that this type of modelling can simulate the crowd behavior in a way very close to realistic situations, whereas specific in vision behavior we considered the internal rotation of an agent when he is alone in addition to external effectives because in real people are self-driven particles.

\section{References}

[1] Sakour, I., Hu, H. (2017). Robot-assisted crowd evacuation under emergency situations: A survey. Robotics, 6 (2) 8 .

[2] Qiu, F., Hu, X. (2010). Modeling group structures in pedestrian crowd simulation. Simulation Modelling Practice and Theory. 18 (2) 190-205.

[3] Van Den Berg, J. (2008). Interactive navigation of multiple agents in crowded environments. in Proceedings of the 2008 symposium on Interactive 3D graphics and games. ACM.

[4] Paris, S., Pettré, J., Donikian, S. (2007). Pedestrian reactive navigation for crowd simulation: a predictive approach. In: Computer Graphics Forum. Wiley Online Library.

[5] Guy, S. J. (2010). Pledestrians: a least-effort approach to crowd simulation. In: Proceedings of the 2010 ACM SIGGRAPH/Eurographics symposium on computer animation. Eurographics Association.
[6] Narain, R. (2009). Aggregate dynamics for dense crowd simulation. ACM transactions on Graphics (TOG). 2009. ACM.

[7] Pelechano, N. (2005). Crowd simulation incorporating agent psychological models, roles and communication. 2005, PENNSYLVANIA UNIV PHILADELPHIA CENTER FOR HUMAN MODELING AND SIMULATION.

[8] Ondrej, J. (2010). A synthetic-vision based steering approach for crowd simulation. ACM Transactions on Graphics (TOG). 29 (4) 123.

[9] Helbing, D., Johansson, A. (2009). Pedestrian, crowd and evacuation dynamics, In: Encyclopedia of complexity and systems science. Springer. p. 6476-6495.

[10] Schadschneider, A. (2011). Evacuation dynamics: Empirical results, modeling and applications, In: Extreme Environmental Events. 2011, Springer. p. 517-550.

[11] Hughes, R. L. (2002). A continuum theory for the flow of pedestrians. Transportation Research Part B: Methodological. 36 (6) 507-535.

[12] Twarogowska, M., Goatin, P., Duvigneau, R. (2014). Macroscopic modeling and simulations of room evacuation. Applied Mathematical Modelling, 38 (24) 5781-5795.

[13] Chraibi, M., Schadschneider, A., Seyfried, A. (2013). On Force-Based Modeling of Pedestrian Dynamics, in Modeling, Simulation and Visual Analysis of Crowds. Springer. p. 23-41.

[14] Helbing, D., Molnar, P. (1995). Social force model for pedestrian dynamics. Physical Review E, 51 (5) 4282.

[15] Abustan, M. S. B. H. (2013). Numerical Simulation of Evacuation Process Against Tsunami Disaster in Malaysia By Using Distinct-Element-Method Based MultiAgent Model.

[16] Henein, C. M., White, T. (2004). Agent-Based Modelling of Forces in Crowds.

[17] Zaharia, M. H. (2009). Agent-based simulation of crowd evacuation behavior. In: WSEAS International Conference. Proceedings. Mathematics and Computers in Science and Engineering. World Scientific and Engineering Academy and Society.

[18] Kiyono, J., Miura, F., Yagi, H. (1998). Simulation of Evacuation Behavior in a Disaster by Distinct Element Method. Vol. 591. 365-378.

[19] Lin, P., Ma, J., Lo, S. (2016). Discrete element crowd model for pedestrian evacuation through an exit. Chinese Physics B. 25(3) 034501.

[20] Mustapha, N. A. B., BADE, B. A. (2011). Collision avoidance series of technique for crowd simulation. Universiti Teknologi Malaysia, Malaysia. 